\title{
Air traffic management based on 4D- trajectories: requirements and practical implementation
}

\author{
Álvaro Rodríguez-Sanz ${ }^{*}{ }^{1}$, Cecilia Claramunt Puchol ${ }^{1}$, Fernando Gómez Comendador ${ }^{l}$, \\ Javier Pérez-Castán ${ }^{I}$, Rosa Arnaldo Valdés ${ }^{l}$, Francisco Serrano Martínez ${ }^{l}$ and Mar Najar \\ Godoy $^{2}$ \\ ${ }^{1}$ Universidad Politécnica de Madrid (UPM), Plaza Cardenal Cisneros N3, Madrid 28040, \\ Spain. \\ ${ }^{2}$ ISDEFE (Ingeniería de Sistemas para la Defensa de España), Calle Beatriz de Bobadilla, \\ N3, Madrid 28040, Spain.
}

\begin{abstract}
The current Air Traffic Management (ATM) functional approach is changing: 'time' is now integrated as an additional fourth dimension on trajectories. This notion will impose on aircraft the compliance of accurately arrival times over designated checkpoints, called Time Windows (TWs). In this context, we review the operational concept of 4D-trajectories, by initially developing an analysis of basic requirements for their implementation in the Communications, Navigation and Surveillance (CNS) systems and then by investigating their management in the future ATM context. We focus on defining the relationships between 4D-trajectories and other concepts and systems of the future ATM framework, and the needs that it will require for its application, detailing the main tools, programs and ATM/CNS systems that must be deployed. We appraise how 4D-trajectories must be managed and planned (negotiation, synchronization, modification and verification processes). Then, based on the degradation of a 4D-trajectory, we define and introduce the necessary corrective measures by evaluating the degradation tolerances and conditions.
\end{abstract}

\section{Introduction}

The recent increase in air traffic demand provides a challenging operational situation for the current European Air Traffic Management (ATM) system [1]. In this way, SESAR (Single European Sky Air Traffic Management Research) and NextGen (Next Generation Air Transportation System) are changing the ATM framework [2], [3]. Achieving accurate prediction of trajectories is a fundamental condition for reliable detection and resolution of conflicts. SESAR and NextGen support the four dimensions (4D) trajectory operational concept, in order to improve efficiency, reliability, sustainability and cost-effectiveness of aircraft operations [2], [4].

\footnotetext{
* Corresponding author: alvaro.rodriguez.sanz@upm.es
} 
The future ATM system relies on the Trajectory Based Operations (TBO) concept. This will require aircraft to follow an assigned 4D-trajectory (time-constrained trajectory) with high precision. TBO involves separating aircraft through a definition of a strategic trajectory (long-term), rather than the currently practicing tactical (short-term) conflict resolution [5]. The main goal is to increase air traffic capacity by reducing the controllers' workload. Nevertheless, real time measures, over the trajectory, will be required to improve reliability and react to unplanned conditions; thereby, maintaining expected capacity [2]. This approach will require aircraft to follow an assigned 4D trajectory with high precision.

The 4D-trajectory concept is based on the integration of time into the $3 \mathrm{D}$ aircraft trajectory. Each point is defined by position (latitude, longitude and flight level) and time. Certain restrictions are currently associated with flight levels. In the future operational framework, it is foreseeable that there will be also restrictions with respect to time [6], [3]. 4D-trajectories will enable a flight to follow a practically unrestricted, optimum trajectory for as long as possible. In exchange, the aircraft will be obliged to meet very accurately an arrival time over a designated point. In the context of TBO, Airspace Users (AUs) will agree a preferred trajectory with Air Navigation Service Providers (ANSPs) and airport operators (AOs). Aircraft and ground systems will exchange information on the trajectory and the expected airspace capacity to ensure that flights comply with the assigned Controlled Time of Arrival (CTA) [6], [3].

To exploit the benefits associated to 4D-trajectories and avoid potential conflicts, aircraft must be kept within very small volumes around their agreed reference trajectory [7]. The main objective is to ensure compliance with the stated separation standards. Thus, it is necessary for 4D-trajectories to be monitored with a high degree of accuracy, precision, data integrity and safety [2]. New tools are essential to allow ATM planners, air traffic controllers (ATCOs), route developers and AUs to obtain the Time Windows (TWs) in which aircrafts are expected to be found at each checkpoint. Accurate TW and trajectory predictions are required for highly efficient ATM procedures [8]. However, this process is strongly influenced by uncertainties [9], [10], such as the actual performance of aircraft and weather/atmospheric conditions affecting the flight. Improved predictability and reliability is one of the eleven target KPAs set in ICAO's Doc 9854 for the Global ATM Operational Concept [5]. It is also one of the requirements of SESAR and NextGen to optimise 4Dtrajectory synchronisation and conflict detection/resolution [2], [3].

\section{Background}

Some of the objectives of SESAR for the future ATM operational concept are to improve efficiency, reliability, sustainability and cost-effectiveness of aircraft operations [2], [4]. The future trajectory management approach is based on a four dimensional framework, composed by the three spatial dimensions and a time constraint [11]. This constrains are called Time Windows (TWs) and require the capability of developing accurate and reliable trajectory predictions [12]. These predictions need to consider external disturbances to the aircraft and internal uncertainty sources [10], [13], [14]. These disturbances and uncertainties may cause a degradation in the trajectory. Thus, trajectory prediction, degradation, and uncertainty management are key elements in the new operation concept of air traffic.

We propose to manage uncertainty with respect to time by establishing several intermediate locations (checkpoints) along a trajectory, where time uncertainty can be constrained by TWs. The methodology for defining TWs uses an aircraft performance model based on EUROCONTROL's Base of Aircraft Data (BADA) family 4.0 [15] and probabilistic approaches to reflect the inherently stochastic nature of air traffic procedures [16], [17]. 
In the context of TBO and RBTs (Reference Business Trajectories), TWs should be sufficiently large to allow AUs and ANSPs to respond flexibly to a variety of flight conditions but sufficiently small to increase certainty and improve capacity. Real-time simulations, done in March and December 2007, with an Airbus A320 investigated 4Dtrajectory management in an en-route environment from both controller and pilot perspectives [18]. The objective was to perform an initial assessment of the operational feasibility of the management and the execution of a RBT and a CTA from a pilot's perspective. Allowing aircraft to adhere to their preferred 4D-trajectories enhanced flight time predictability, but impacted controllers' tasks [18]. Another experiment conducted by EUROCONTROL in 2012, with an Airbus A320 test aircraft that flew from Toulouse to Stockholm, established a tolerance window of between -2 minutes and +3 minutes over the route and \pm 30 seconds for CTA [11]. In addition, pilots were exposed to situations where the aircraft was deviated from the planned path. The results showed that adherence to 4Dtrajectories was feasible in cruise phase while the TW for CTA was more difficult to achieve and required increased coordination between pilots and controllers [11].

The TBO concept for the future ATM system encompasses 3 types of trajectories [2], [4]: (a) Business Development Trajectory (BDT) (it consists of the initial part of the planning process, which could occur even years before the operation, whose purpose is the definition and programming of the resources and institutions that are going to be involved); (b) Shared Business Trajectory (SBT) (the trajectory that will be published in order to carry out a collaborative planning process with all stakeholders); and (c) Reference Business Trajectory (RBT) (the trajectory that airlines agree to fly, and that navigation service providers agree to facilitate).

The objectives of this study are to: (a) review the operational concept of 4Dtrajectories, by initially developing an analysis of basic requirements for their implementation in the field of CNS/ATM concepts and systems; (b) investigate how to manage the 4D-trajectories and the phases of the process; and (c) analyse a 4D-trajectory evolution and degradation to define and introduce the necessary corrective measures through tolerance windows.

\section{Methodology}

Initially, we focus on analysing the relationships between 4D-trajectories and other concepts and systems of the future ATM framework, and the needs that its implementation will require.

\subsection{Relationship with other CNS/ATM concepts and systems}

Firstly, 4D-trajectories rely on previous operational steps to check their viability [1], [2], [11]: ID4 (Initial 4D Trajectory Management); FRA (Free Route Airspace and Point Merge. Secondly, three procedural concepts [1], [2]: CDO and CCO (Continuous Descent/Climb Operations), UDPP (User Driven Prioritization Process) and STAMs (Short-Term ATFCM -Air Traffic Flow and Capacity Management-Measures) will adjust the aircraft path to the negotiated reference trajectories whenever is possible. Lastly, for the implementation of 4D-trajectories it is essential to exchange updated and accurate information during all flight phases [1], [2], using SWIM (System Wide Information Management). Moreover, planning of operations should be achieved through a collaborative decision-making process among all stakeholders, including A-CDM (Airport Collaborative Decision Making) [1], [2].

The systems or tools framework can be related to their functionality regarding 4D operations [1], [2]: flight sequencing and management systems, planning and trajectory 
prediction systems, ATFCM (Air traffic flow and capacity management) systems, flight surveillance and monitoring systems, communication systems; and Flight Management System (FMS) and Human-Machine Interface (HMI) systems.

\subsection{D-Trajectory management}

Now, we appraise how 4D-trajectories must be managed and planned, defining the negotiation, synchronization, modification and verification processes.

4D trajectory management goes through several phases. The first step is the planning phase, when the trajectory is treated as SBT and the process of trajectory negotiation occurs. Then, when it is approved by all stakeholders, it is transferred to RBT. Finally, the execution phase arises, which involves the process of trajectory monitoring. The monitoring includes the analysis and, if it is necessary, the modification of the RBT. These activities are composed of the verification, updating, review and synchronization processes of the trajectory. Figure 1 represents the processes of 4D-trajectories management.

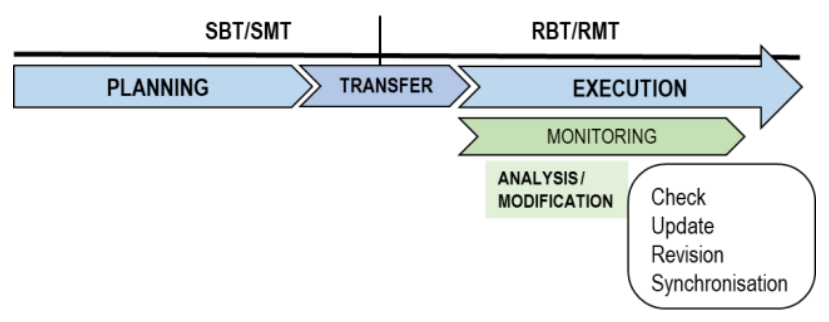

Fig. 1. 4D-Trajectory management processes.

\subsection{Degradation tolerance windows}

After the theoretical analysis, we propose the degradation tolerances and conditions, under which 4D-trajectories must be updated.

In this study, 4D-trajectories are modelled using a point mass model and the EUROCONTROL's BADA (Base of Aircraft Data) methodology [15]. The variability of parameters that influence the definition and development of 4D-trajectories is appraised stochastically through Monte Carlo simulation, by considering different scenarios and several checkpoints. The Monte Carlo method allows us to obtain a large number of simulations (potential trajectories) by varying the influential parameters. The results reflect the stochastic nature of the evolution of the flight by including actual uncertainties in trajectory prediction. Once the simulations have been performed, we can define the expected TW at each checkpoint of the agreed trajectory, thereby, providing airspace users, ANSPs and airspace operators with a framework for traffic synchronization and conflict resolution [17]. The size of a TW on a Reference Business Trajectory (RBT) should at least represent the time interval within which any aircraft arriving at the checkpoint can avoid collisions with other aircraft [19]. To estimate the TWs, we start by setting the position (x) of the checkpoints (CP), as shows Figure 2. Each aircraft must hit them within a specified time interval [9].

We propose a flexible approach in which TWs can be distributed along the entire 4Dtrajectory of a flight in such a way ATCOs can manage the punctuality of aircraft as they transit between sectors and also along their entire trajectory. To illustrate the method for calculating TWs we define ten checkpoints along the three flight phases of the proposed scenario (climb, cruise, descent). 
As shown in Figure 2, Checkpoint (CP) 1 is located in the middle of the ascent phase and CP 2 at the end of it, at 75 NM and 151 NM, respectively; CPs 3, 5 and 7 are located in the middle of the horizontal flight sections on cruise phase, at $200 \mathrm{NM}, 303 \mathrm{NM}$ and 409 $\mathrm{NM}$, respectively; CP 4 is located in the intermediate point of the ascending flight (flight level change between FL360 and FL380), at 253 NM; CP 6. which is placed like CP4, but in the descending flight level change, is located at $357 \mathrm{NM}$; and finally, CP 9 and CP 10 are in the middle and at the end of the descent phase, respectively, at $459 \mathrm{NM}$ and $509 \mathrm{NM}$. Each flight phase is treated as independent when it comes to the calculations. This approach allows us to work with "blocks" with different characteristics (ascent, cruise, flight level change, descent), and replicate other potential flight structures by combining them.

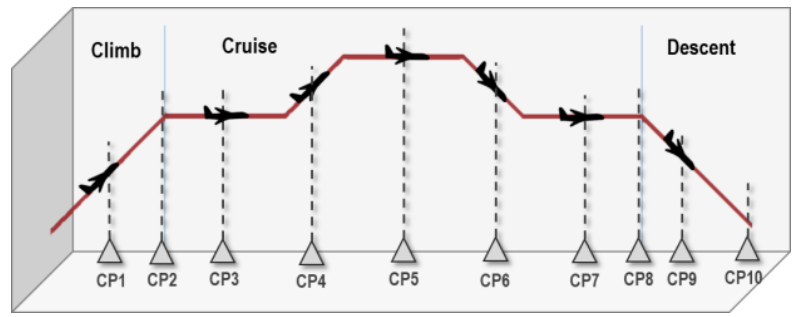

Fig. 2. Waypoints distribution through the flight.

After obtaining the arrival times at each CP by the 1500 Monte Carlo simulations, we can represent a histogram that can be modelled as a normal distribution for each CP. For example, for CP 5, we obtained a normal distribution centred in $\mu=1194$ seconds from the start of the segment and a standard deviation of $\sigma=4.9$ seconds. Both a K-S test and $\chi^{2}$ goodness-of-fit test were used to ensure the "power" of curve fitting [20]. Fitted probability curves for the arrival times at each $\mathrm{CP}$ are given by the probability density function: e.g. fitted probability curves for the arrival times at CPs 5 and 7 are shown in Figure 3 by the probability density function:

$$
f_{\text {NORMAL }}(t, \mu, \sigma)=\frac{1}{\sqrt{2 \pi} \cdot \sigma} \cdot e^{-\left(\frac{t-\mu}{2 \sigma}\right)^{2}}
$$

By setting the position ( $\mathrm{x}$ ) of the checkpoint and fitting time variation to a normal distribution we can define different TWs or time intervals depending on the accuracy required. The width of the TW is an indicator of how predictable a flight is. Setting longer intervals increases predictability and reduces uncertainty but leads to less efficient time management. Due to it, we chose to estimate the time window with $2 \sigma$, that is, with a confidence level of $95.46 \%$. This means that the aircraft will be in each checkpoint at a certain time with a probability of $95.46 \%$.
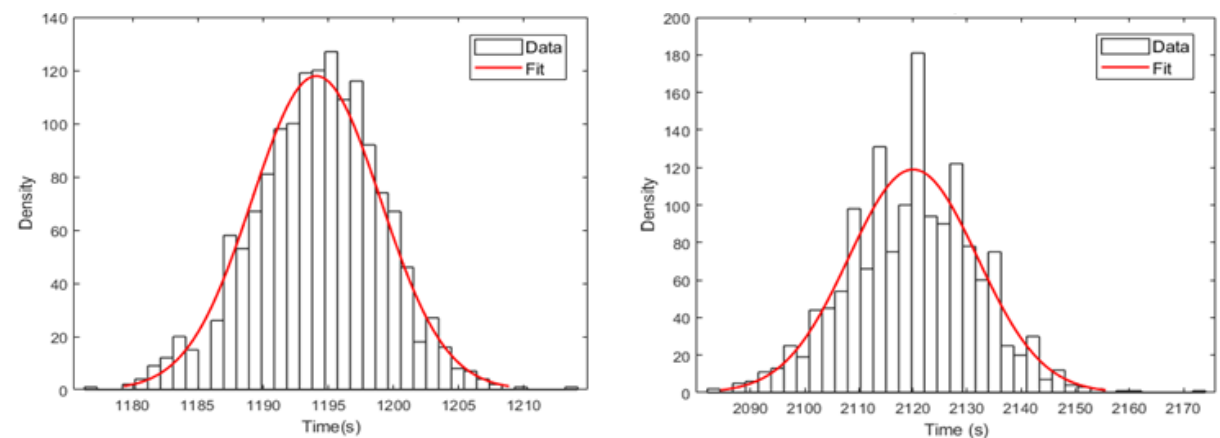

Fig. 3. Statistical distribution of the aircraft's arrival time in seconds at CP 5 (left) and CP 4 (right). 
Therefore, there is a $95.46 \%( \pm 2 \sigma)$ probability that an aircraft will be at CP 5 (303 NM) within a TW of \pm 10 seconds centred at 1194 seconds. Similarly, the arrival times at CP 7 (409 NM) can be fitted to a normal distribution with mean $\mu=2120$ seconds and a standard deviation of $\sigma=12$ seconds. There is a $95.44 \%$ probability that an aircraft will reach CP 4 within a TW of $\pm 24 \mathrm{~s}$. The values of $\sigma$ are higher for CP 4 than for CP 3 . This means that the further the distance (progress of a flight) the greater the uncertainty and data dispersion. Table 1 gives the mean $(\mu)$ and the standard deviation $(\sigma)$ of each TW (measured in seconds). The standard deviation is a measure of dispersion about the mean data that enable us to define the different tolerance windows at each point.

Table 1. Time Windows values for CP.

\begin{tabular}{c||c|ccc} 
Flight Phase & Checkpoint & $\boldsymbol{\mu}(\mathbf{s})$ & $\boldsymbol{\sigma}(\mathbf{s})$ & Time Window (s) \\
\hline \multirow{2}{*}{ Ascent } & $\mathbf{1}$ & 777 & 9.95 & \pm 20 \\
\cline { 2 - 5 } & $\mathbf{2}$ & 1353 & 47.9 & \pm 96 \\
\hline \multirow{5}{*}{ Cruise } & $\mathbf{3}$ & 393 & 0.95 & \pm 2 \\
\cline { 2 - 5 } & $\mathbf{4}$ & 864 & 2.99 & \pm 6 \\
\cline { 2 - 5 } & $\mathbf{5}$ & 1194 & 4.9 & \pm 10 \\
\cline { 2 - 5 } & $\mathbf{6}$ & 1664 & 8.2 & \pm 17 \\
\cline { 2 - 5 } & $\mathbf{7}$ & 2120 & 11.9 & \pm 24 \\
\hline \multirow{2}{*}{ Descent } & $\mathbf{8}$ & 2376 & 14.1 & \pm 29 \\
\cline { 2 - 5 } & $\mathbf{9}$ & 478 & 2.7 & \pm 6 \\
\hline
\end{tabular}

It is recalled that the three phases have been analysed separately, so the TWs are not cumulative from one flight phase to another.

Based on these results, we propose thresholds or tolerance windows, which will serve as a margin of degradation for the aircraft and will set the necessary updates. That is, in case of exceeding these tolerance windows, the RBT must be recalculated and updated. Furthermore, these values will be justified together with the minimum separations between aircraft stipulated by ICAO [21].

\section{Results}

Taking the values shown in Table 1 as a guide along with other assessments, such as the flight phase in which each CP is located, new tolerance windows will be established (time tolerances for the 4D-trajectory). The objective is that any aircraft can take these tolerance values as a reference for the RBT's update (in the case of non-compliance of the time requirements). Table 2 shows the tolerance windows proposed for each CP. Flight phases are treated as independent segments, so windows are not cumulative from one phase to another. Trajectory degradation increases when crossing the different CPs, although in each phase it is restarted. This is because an individual calculation has been made for ascent, cruise and descent, using independent codes in the MATLAB simulations [23].

We consider windows of 20 and 96 seconds for the ascent phase. We decided to increase these values compared to values shown in Table 1 because the ascent phase entails a great associated uncertainty due to meteorology (wind) and airlines procedures policy. In the cruise phase, the points located after a horizontal stabilized flight have a tolerance window of \pm 5 seconds with respect to the target time of arrival (overflight time) and the stretches of flight level changes have a tolerance of \pm 15 seconds. In the descent phase, although the end of the descent, according to the results of TWs, the degradation is much lower $( \pm 13 \mathrm{~s})$ there is a greater margin $( \pm 50 \mathrm{~s})$. This is due to the uncertainty that this phase contains. As in the ascent phase, this stage is influenced by the airlines procedure policies 
too (depends on their targets: to pollute less, to reduce flight time, etc.) and the conditions of the arrival airport (if it is congested or with special conditions like low visibility, etc.).

Table 2. Tolerance Window for each flight phase.

\begin{tabular}{c||c|c} 
Flight Phase & Checkpoint & Tolerance Window (s) \\
\hline \multirow{2}{*}{ Ascent } & $\mathbf{1}$ & \pm 30 \\
\cline { 2 - 3 } & $\mathbf{2}$ & \pm 100 \\
\hline \multirow{4}{*}{ Cruise } & $\mathbf{3}$ & \pm 5 \\
\cline { 2 - 3 } & $\mathbf{4}$ & \pm 20 \\
\cline { 2 - 3 } & $\mathbf{5}$ & \pm 25 \\
\cline { 2 - 3 } & $\mathbf{6}$ & \pm 40 \\
\hline \multirow{2}{*}{ Descent } & $\mathbf{7}$ & \pm 45 \\
\cline { 2 - 3 } & $\mathbf{8}$ & \pm 50 \\
\cline { 2 - 3 } & $\mathbf{9}$ & \pm 15 \\
\hline
\end{tabular}

The established windows will be maintained regardless of whether they have been met in the previous CP or not. That is, if the RBT has to be updated because the aircraft does not meet the target time of arrival at one $\mathrm{CP}$, the overflight time for this point will change, but not the TW in which it has to pass.

Finally, we validated these restrictions with real flights using the EUROCONTROL's NEST tool [22], comparing its initial flight plan and their actual flight. Initial results (validated at an intra-European route) show that for the aircraft type Boeing B737, the proposed TW model can control the time tolerance within less than 27 seconds along the passing CPs of an en-route segment.

\section{Conclusions}

Both SESAR and NextGen programs seek to expand and improve the operational framework of TBOs. The key point of these projects is the evolution in trajectory management towards "gate to gate" operations, providing agile, optimized, aircraft-specific routing to meet service provider gate-to-gate flow management constraints. This translates into greater freedom for AUs to establish their optimal routes while considering flight as a continuous event that minimizes the number of people involved in its correct development from planning to post-operational analyses.

After the analysis of processes, phases and implications related to 4D-trajectory management (operational concepts and technologies), it is extracted that, together with the necessary optimization in the ATCO-pilot communications, a very high level of automation is required regarding the processes of verification, update, review and synchronization of BDT, SBT and RBT. We have appraised a 4D-trajectory evolution to establish the points and conditions for updating the RBTs. This has been approached through the concept of Time Windows. The definition of tolerance time windows over the trajectory (at different checkpoints) provide a framework for reviewing the RBT. By adopting these controlled times for a flight, ATCOs and pilot would have to renegotiate the trajectory if those tolerances are not fulfilled.

The main contribution of this work is the provision of a holistic vision of the systems and concepts that will be necessary to implement the new 4D-trajectory concept efficiently, thus enhancing performance. We also propose tolerance windows for trajectory degradation, to understand both when an update is necessary and what are the conditions required for pilots and ATCOs to provide this update. 4D-trajectories will improve traffic synchronization, while its adequate management will potentially ease conflict resolution. These are cornerstones in future airspace operational environments. 


\section{References}

[1] SESAR, "European ATM Master Plan - Edition 2015," Luxembourg: Publications Office of the European Union, 2015.

[2] SESAR, "SESAR Concept of Operations Step 2 Edition 2014 (Ed. 01.01.00)," Brussels: SESAR Joint Undertaking, 2014.

[3] NextGen, "Concept of Operations for the Next Generation Air Transportation System. Version 3.2," Washignton D.C.: NextGen, 2011.

[4] FAA, "The Future of the NAS," Washington D.C: U.S. Department of Transportation, Federal Aviation Administration, Office of NextGen, 2016.

[5] ICAO, "Doc 9854: Global Air Traffic Management Operational Concept," Montreal: International Aviation Civil Organization, 2005.

[6] SESAR, "SESAR Concept of Operations Step 1 Edition 2012 (Ed. 01.00 .00 )," Brussels: SESAR Joint Undertaking, 2012.

[7] CATS, "Contract-Based Air Transportation (CATS) Concept of Operation D1.2.2," Brussels: European Organisation for the Safety of Air Navigation, 2010.

[8] F. Han, B. L. W. Wong, and S. Gaukrodger, "Pinch-and-pull with spatial-temporal energy trajectory: New display concept for in-flight energy management," Aircr. Eng. Aerosp. Technol., vol. 85, no. 2, pp. 126-135, 2013.

[9] K. Margellos and J. Lygeros, "Toward 4-D Trajectory Management in Air Traffic Control: A Study based on Monte Carlo Simulation and Reachability Analysis," IEEE Trans. Control Syst. Technol., vol. 21, no. 5, pp. 1820-1833, 2013.

[10] E. Casado, C. Goodchild, and M. Vilaplana, "Identification and Initial Characterization of Sources of Uncertainty Affecting the Performance of Future Trajectory Management Automation Systems," in Proceedings of the 2nd International Conference on Application and Theory of Automation in Command and Control Systems (ATACCS'2012), 2012, pp. $170-175$.

[11] L. H. Mutuel, P. Neri, and E. Paricaud, "Initial 4D Trajectory Management Concept Evaluation," in Tenth USA/Europe Air Traffic Management Research and Development Seminar, 2013.

[12] T. Rentas, S. Green, and K. Cate, "Characterization Method for Determination of Trajectory Prediction Requirements," in 9th AIAA Aviation Technology, Integration, and Operations Conference (ATIO), Aviation Technology, Integration, and Operations (ATIO) Conferences, 2009.

[13] T. Pabst, T. Kunze, M. Schultz, and H. Fricke, "Modeling external disturbances for aircraft in flight to build reliable 4D trajectories," in 3rd International Conference on Application and Theory of Automation in Command and Control Systems (ATACCS'2013), 2013.

[14] J. L. Garcia-Chico, R. A. Vivona, and K. T. Cate, "Characterizing Intent Maneuvers from Operational Data-A Step towards Trajectory Uncertainty Estimation," in AIAA Guidance, Navigation and Control Conference and Exhibit, 2008.

[15] EUROCONTROL, "User manual for the Base of Aircraft Data (BADA) Family 4. EEC Technical Scientific Report No. 12/11/22-58.," Brussels: European Organisation for the Safety of Air Navigation, 2014.

[16] E. Koyuncu, M. Uzun, and G. Inalhan, "Cross-entropy-based cost-efficient 4D trajectory generation for airborne conflict resolution," Proc. Inst. Mech. Eng. Part G J. Aerosp. Eng., vol. 230, no. 9, pp. 1605-1631, 2016.

[17] Á. Rodríguez-Sanz et al., "4D-trajectory time windows: definition and uncertainty management,” Aircr. Eng. Aerosp. Technol., vol. 91, no. 5, pp. 761-782, 2019.

[18] EUROCONTROL, 4D Trajectory management: an initial pilot perspective. Brussels, 2008.

[19] F. Han, B. L. W. Wong, and S. Gauhrodger, "Improving future air traffic punctuality: 'pinchand-pull' target windows," Aircr. Eng. Aerosp. Technol., vol. 82, no. 4, pp. 207-216, 2010.

[20] G. D. Garson, Curve Fitting and Nonlinear Regression. Asheboro, NC: Statistical Associates Publishers, 2012.

[21] OACI, "Doc. 4444 Gestión del tránsito aéreo," 2007.

[22] EUROCONTROL, "NEST modelling tool." 2017.

[23] MathWorks, "MATLAB," www.mathworks.com/products/matlab. 2017. 